\title{
Les études des outils et médias éducatifs : une approche composite
}

Frédéric Marty

\section{OpenEdition}

1 Journals

Édition électronique

URL : http://journals.openedition.org/communicationorganisation/4223

DOI : 10.4000/communicationorganisation.4223

ISSN : $1775-3546$

Éditeur

Presses universitaires de Bordeaux

Édition imprimée

Date de publication : 1 juin 2013

Pagination : 205-212

ISBN : 978-2-86781-883-7

ISSN : 1168-5549

Référence électronique

Frédéric Marty, «Les études des outils et médias éducatifs : une approche composite », Communication et organisation [En ligne], 43 | 2013, mis en ligne le 01 juin 2015, consulté le 19 avril 2019. URL : http://journals.openedition.org/communicationorganisation/4223 ; DOI : 10.4000/ communicationorganisation. 4223 


\title{
Les études des outils et médias éducatifs : une approche composite
}

\author{
Frédéric Mortụ
}

Pour Jacques Perriault et Dominique Wolton, l'enjeu des Sciences de l'Information et de la Communication est de tenir en cohabitation l'observation « des instruments et leurs usages, des pratiques fonctionnelles et symboliques, des langages, des dispositifs, des circulations d'information et de savoirs, des polémiques, des stratégies et des politiques » (Perriault, 2010). L'observation des outils et médias éducatifs, qu'elle se situe en amont ou en aval de leur production, n'échappe pas à cette ambition.

L'objet de cet article est de revenir sur ces différents éléments à articuler dans le cadre de la réflexion qui est la nôtre sur l'audiovisuel éducatif à l'ère numérique ${ }^{2}$. Par extension, cette articulation nous permet de conceptualiser notre objet de recherche et de cerner l'approche la plus à même de servir notre questionnement. Pour ce faire, nous mettrons en perspective les différents travaux ayant eu les outils et médias éducatifs comme objet. Pour Alain Jaillet (Depover, 2009), on distingue ceux qui se concentrent sur les phénomènes éducatifs, dont l'essentiel des travaux relèvent des Sciences de l'Éducation ; les recherches en Sciences de l'Information et de la Communication étudiant des phénomènes davantage liés aux dimensions sociales; enfin, un important secteur s'articulant autour des questions posées sur un volet informatique, avec la communauté Environnement Informatique pour l'Apprentissage Humain (EIAH), qui, bien que comptant des représentants de différents horizons, est principalement animée par les informaticiens. Nous tenterons ici de dépasser ces oppositions disciplinaires, d'autant que depuis les années 1960, nous assistons à la structuration de ce que certains nomment une «interdiscipline » (Charlier et Peraya, 2007). Si cela peut parfois induire une certaine confusion quant aux délimitations de ce champ de recherche, il n'en demeure pas moins que ce dernier est marqué par une structuration transdisciplinaire

1 Doctorant, Université de Toulouse, LERASS (EA 827) ; fmarty@univ-tlse2.fr

2 L'état de l'art proposé ici s'inscrit dans notre travail de thèse portant sur les usages enseignants du site.tv, service de vidéo éducative à la demande. www.lesite.tv 
manifeste : ce qui consiste, pour Patrick Charaudeau, « à établir de véritables connexions entre concepts, outils d'analyse et modes d'interprétation de différentes disciplines " (Charaudeau, 2010). Nous avons choisi de regrouper ces différents travaux autour de quatre thèmes, selon qu'ils sont centrés sur l'apprentissage, les contenus, la technologie ou le dispositif. Même si ces catégories offrent une hétérogénéité au regard des disciplines académiques qu'elles recoupent (sciences de l'éducation, sciences de l'information et de la communication, sociologie, psychologie, etc.), nous verrons que l'approche communicationnelle est assez circonscrite. En conclusion, nous reviendrons sur les enjeux propres à notre travail sur l'audiovisuel éducatif à l'ère numérique, et la façon dont il s’inscrit dans ce champ.

\section{Les approches centrées sur l'apprentissage}

Nous avons regroupé ici plusieurs démarches que Jacques Wallet distinguait entre approches centrées sur l'« apprenant » et l'« accompagnement » (Depover, 2009, 15-24). Elles relèvent principalement des Sciences de l'Éducation, et portent leur attention sur l'efficacité des outils et médias éducatifs. On se demande donc si certaines technologies vont avoir un effet, ou non, sur l'apprentissage et les formes qu'il revêt. Ce type d'approches privilégie des inspirations nomothétiques, visant à établir des lois générales, au détriment de recherches qualitatives. Elles se sont d'autant plus développées que la technologie numérique permet de quantifier plus en détail certaines actions. C'est ce que Pierre Moeglin reproche en critiquant une "superposition du quantitatif et des métries» (Moeglin, 2005, 25). Alors qu'il s'agit pour nombre de ces études de quantifier et d'évaluer l'efficacité de ces technologies éducatives, force est de constater qu'il n'en ressort pas pour autant de réponse univoque. Cette approche dite de la « technologie éducative " (années 1960), a en quelque sorte "matricé » ce champ de recherche, et doit être mise en regard des recherches américaines du courant fonctionnaliste. En effet, ces chercheurs ne visent pas autre chose qu'une étude scientifique des incidences sociales des médias et de la communication en général, à travers l'exemple des technologies éducatives. Du côté des études centrées sur l'apprenant on retrouve plusieurs inspirations théoriques relevant de la psychologie. Par exemple, en Amérique du nord, les influences du behaviorisme se manifestent à travers l'écho que leur offre Burrhus Frederic Skinner, en conférant aux outils et médias éducatifs le rôle de stimuli, et l'usage de l'ordinateur à l'école constitue un terrain propice aux théories cybernétiques proposées par Norbert Wiener. Dans un tel modèle, la place de l'enseignant est mise en question, entre développement de nouvelles compétences et substitution à un nouvel interlocuteur, dans un schéma éducatif repensé (i.e. enseignement à distance).

C'est le paradigme expérimental qui domine alors à cette époque, mais on observe progressivement un décentrement méthodologique. Il n'est plus seulement question d'évaluer un progrès supposé, mais de chercher 
à comprendre et expliquer l'efficacité des technologies éducatives. Cette évolution du raisonnement traduit le passage d'une démarche déductive à une démarche inductive. Pour Georges-Louis Baron et Éric Dané (Depover, 2009), la tension entre ces deux types d'approches demeure permanente entre le paradigme expérimental dont le modèle prototypique de «l'expérimentation randomisée » est censé apporter des informations de nature causale, et d'autre part, les approches qualitatives fondées sur des études de cas et des méthodes de type ethnographique, visant moins à valider des théories qu'à expliquer ce qui advient.

\section{Les approches centrées sur les contenus}

Au même moment où se développent les premières approches cherchant à évaluer l'efficacité des outils et médias éducatifs, un courant s'intéresse plus particulièrement aux contenus véhiculés par ces derniers. Dès les années 1960, il se développe aux côtés du mouvement sémiologique, au sein du Centre d'etudes de communication de masse (CECMAS), avec notamment Roland Barthes. On y retrouve des personnes comme Christian Mertz qui, par ailleurs, œuvrent à la diffusion de "l'esprit sémiologique " auprès des enseignants dans le cadre d'initiatives comme l'ICAV (Initiation à la Culture AudioVisuelle), lancée en 1966 dans l'Académie de Bordeaux. Ces expériences attestent d'une certaine filiation avec les travaux menés par Henry Dieuzède, ou Michel Tardy, pour qui la pédagogie des messages visuels exige d'abord « une réflexion sur le statut de l'image et ses coordonnées ontologiques». Ils contribuent à mettre en évidence l'existence d'une "instance discursive spécifique aux discours audiovisuels à finalités éducatives : le didactique filmique "(Jacquinot, 2007, 174). Ce questionnement est aussi celui des premiers travaux de Geneviève Jacquinot et celui du premier pont entre deux nouvelles disciplines fraîchement instituées : les Sciences de l'Éducation (1967) et les Sciences de l'Information et de la Communication (1972).

La réflexion autour des contenus de cette éducation " par » les médias, se porte également sur les finalités d'une telle démarche. Le développement des médias de masse, comme la télévision, et la massification du système éducatif interpellent chercheurs et militants de l'éducation populaire sur le potentiel de « démocratisation » des outils et médias éducatifs. On parle alors d'une éducation « avec », puis « aux » médias, comme un nouvel espace de citoyenneté à conquérir (Gonnet, 2001). La France est d'ailleurs marquée par une institutionnalisation précoce de cette ambition avec la création du Centre de Liaison entre l'Éducation et les Médias d'Information (CLEMI) en 1983. L'appropriation de l'objectif de " pensée critique " véhiculée par les discours sur l'éducation aux médias est toutefois hétérogène (Piette, 1969). Plus récemment, les débats autour de la notion de " médiaculture » posent de nouveau la question des contenus de l'éducation aux médias (Jacquinot, 2011) en décentrant les objets de cette éducation vers les pratiques culturelles 
effectives des jeunes notamment : séries télé, jeux vidéos, réseaux sociaux, etc. (Jacquinot, 2011).

\section{Les approches centrées sur la technologie}

Nous regroupons ici les différentes recherches ayant pour objet de départ la technologie en tant que telle. Cela passe par l'étude des conditions dans lesquelles sont conçus ces outils et médias éducatifs, la mesure de leur diffusion, ou l'analyse de leur évolution. Ces différentes approches "technologiques" sont également abordées de façon transdisciplinaire.

Pour Brigitte Albero, parmi les grands courants d'étude, on peut mettre en évidence ceux qui se penchent sur la conception des environnements techniques (Albero, 2004). Ces derniers s'intéressent aux principes de conception, au développement et à l'évaluation de produits (outils, logiciels, langages, etc.) utiles à l'apprentissage. Cet intérêt cohabite avec celui de disciplines comme l'informatique qui dès les années cinquante ont eu tendance à réunir informaticiens, mathématiciens, psychologues, neurophysiologistes, anthropologues et physiciens autour de la cybernétique ou encore de l'intelligence artificielle. On peut voir un prolongement et une concrétisation de ce travail commun à travers la constitution de ce que l'on appelle de nos jours l'étude des Environnements Informatiques pour l'Apprentissage Humain (EIAH). Cette collaboration entre psychologues, didacticiens ou ergonomes témoigne d'un dépassement de la question " technique » pour interroger l'utilisabilité ou encore l'usage de cette dernière.

Le développement de la radio, puis de la télévision éducative dans les années soixante, de l'enseignement informatique dans les années soixante-dix, du multimédia et de la formation à distance dans les années quatre-vingt, le développement des Espace Numériques de Travail (ENT) et du e-learning dans les années 2000, ont à chaque fois été accompagnés d'études cherchant à mesurer leur diffusion. L'évaluation des investissements publics en la matière explique notamment la multiplication de ces évaluations nationales et internationales ne mettant pas forcément en perspective les résultats quantitatifs obtenus. Sans pour autant revendiquer un cloisonnement entre les différents outils et médias éducatifs, les approches " archéologiques » ont elles aussi la technique comme angle d'étude. On peut donc trouver un certain nombre de recherches portant respectivement sur la radio scolaire, la télévision éducative, les images lumineuses, etc. Mais, comme l'atteste le colloque Pour une histoire de l'audiovisuel éducatif (1950-2000) (Pastre et al., 2007), ces différentes études s'inscrivent tout aussi largement dans l'approche " dispositive », véritable « carrefour » des travaux portant sur les outils et médias éducatifs (Moeglin, 2010). 


\section{Les approches centrées sur le dispositif}

Si pour Bernard Miège, «les approches pédagogiques, techno-expérimentales et même cognitivistes [sont] encore largement dominantes, sinon parmi les chercheurs, du moins chez les professionnels » (Miège, 2004), il n'en demeure pas moins que depuis les années 1980 les approches centrées sur le dispositif ont permis un certain rééquilibrage. En effet, des chercheurs comme Geneviève Jacquinot, qui est à l'initiative de cette catégorisation, proposent " une conception élargie du dispositif de formation (qui) s'élabore au carrefour de la théorie piagétienne de l'apprentissage par les méthodes actives, de la psychologie cognitive et de courants relevant des sciences de l'information et de la communication inspirés des travaux de Michel de Certeau [...] attentive à la diversité des formes d'appropriation, $\mathrm{y}$ compris à celles qui procèdent par détournement et braconnage » (Moeglin, 2010). La présence de Geneviève Jacquinot au centre de ce carrefour n'est pas surprenante puisqu'elle a contribué aux premières démarches réflexives sur la structuration théorique de ce champ de recherche (Jacquinot, 1985). En s'ouvrant à la notion de dispositif cette approche s'ouvre également à celles de médiation et d'interactivité.

Parmi ces approches, qui intègrent notamment la question de l'usage dans leurs postulats, nous pouvons citer les travaux de Jacques Perriault. Son ouvrage sur la « logique de l'usage » insiste sur la volonté d'inscrire les contextes d'utilisation dans une histoire des techniques, tout en révélant la grande diversité des usages et les différents facteurs qui les construisent (Perriault, 2008). Pour lui, cette démarche s'applique à l'ensemble des " machines à communiquer » qui, " numériques ou non, [...] produisent et transmettent en effet des simulacres, ce qui nous interdit de les dissocier, du point de vue de l'usage, des dispositifs antérieurs, tels que la lanterne magique, téléphone et autres appareils audiovisuels »(Perriault, 2002). Cette approche par les usages permet donc une approche transdisciplinaire, comme par exemple les travaux portant sur les " conditions des genèses d'usages des technologies numériques dans l'éducation "(Assude et al., 2010). À cet instant nous pouvons également citer les travaux poursuivant les réflexions du séminaire «Industrialisation de la Formation » (créé en 1991). Partant du constat qu'il manque à de nombreuses études « une approche sociale, culturelle, politique, institutionnelle, idéologique du statut éducatif de ces outils et médias » (Moeglin, 2005, 11), ils proposent un paradigme alternatif. Cette approche par « l'industrialisation éducative » (Moeglin, 2010) reproche notamment aux approches des technologies éducatives, à travers la notion d'efficacité, d'être " marquées économiquement et idéologiquement»(Chaptal, 2004, 209). Toutes ces approches ont également en commun de ne pas vouloir limiter les outils et médias éducatifs à leur caractère éducatif, c'est d'ailleurs pour cela qu'ils récusent la terminologie de TICE (Technologie de l'information et de la communication pour l'éducation) et celle de «technologie éducative », vue comme une simple " panoplie». 


\section{Pour une approche composite de l'audiovisuel éducatif numérique}

Finalement, c'est bien le " point de vue qui crée l'objet ", comme le souligne Bruno Ollivier avec les mots de Ferdinand de Saussure (Davallon, 2004). Or, l'approche communicationnelle se distingue par sa capacité à construire " un objet scientifique (une représentation homogène, cohérente, complète et partagée) à partir d'un ensemble d'objets concrets de statut et de nature différents dont certains sont des objets techniques " (Davallon, 2004). L'approche composite à laquelle nous invite Joëlle Le Marec fait sens ici : de la construction de l'objet de recherche à la pratique de terrain qu'elle induit. Les nombreuses approches que nous avons évoquées auparavant conduisent également à penser que les analyses « technocentrées », tout comme les analyses " ethnocentrées », ont montré leurs limites si elles sont isolées (Depover, 2009, 15-16). Pour rendre compte au mieux de la complexité de notre objet d'étude, notre ambition méthodologique doit être plurielle. En choisissant de traiter de l'audiovisuel éducatif à l'ère numérique ainsi que de ses usages par les enseignants, nous inscrivons notre approche des outils et médias éducatifs sous un angle résolument communicationnel (Moeglin, 2005).

Il s'agit donc de positionner notre objet numérique, lesite.tv, dans sa lignée technique, sa logique d'usage et le paradigme d'industrialisation éducative qui l'entoure. Une fois cet effort de contextualisation entrepris, il s'agira d'accéder à notre objet par l'intermédiaire de ses usages (Jouët, 2011), envisagés ici comme " les phénomènes par lesquels se construisent et se manifestent les rapports aux objets techniques »(Le Marec, 2002). Rappelons que lesite.tv, avec plus de 3000 vidéos accessibles en téléchargement ou en streaming, et plus de 100000 enseignants abonnés dans plusieurs milliers d'établissements, est aujourd'hui le premier site français de vidéos éducatives. Sa création remonte à 2003 et au rapprochement entre France 5 et le CNDP qui créent un groupement d'intérêt économique (GIE). Une approche strictement quantitative ne nous donnerait pourtant pas la mesure de ces usages et de ses non-usages. En effet, au regard des expériences précédentes d'audiovisuel éducatif (lanterne magique, cinéma scolaire, télévision éducative, etc.) la prudence doit être de mise face aux partis-pris tantôt apologétiques et tantôt apocalyptiques qui jalonnent l'utilisation de l'audiovisuel en classe : c'est tout l'intérêt d'un « retour » aux usagers. Mais, pour éviter un certain positivisme et écarter les risques d'une prise trop forte à l'air du temps, il est important de convoquer les aspects symboliques, sociaux et techniques, et de replacer l'usager et l'interprétation de ses usages au croisement des tensions qui l'animent grâce à une série d'entretiens compréhensifs. C'est en ce sens qu'une approche communicationnelle permet de rendre compte de la complexité du rapport entre médias et école. En d'autres termes, c'est « penser l' «objectivité» des outils et médias éducatifs dans la dialectique réflexive de la subjectivation éducative et de l'objectivation communicationnelle » (Moeglin, 2005, 30). 
Pour conclure, nous souhaitons revenir sur la démarche qui a été la nôtre pour cet article. En ayant choisi de ne pas limiter notre catégorisation aux questions disciplinaires, nous avons souhaité montrer avec plus de dynamique l'importance à accorder à la formulation de l'objet de recherche. De ce fait, nous avons mis côte à côte certains travaux de sorte que notre approche éclaire sous un autre jour les états de l'art menés par ailleurs (Jacquinot, 2007 ; Depover, 2009 ; Moeglin, 2010), d'un point de vue disciplinaire ou chronologique. Cet exercice de synthèse est forcément partiel ; nous espérons qu'il nourrira néanmoins le dialogue, souvent fertile, autour des outils et médias éducatifs.

\section{BIBLIOGRAPHIE}

ALBERO B., «Technologies et formation: travaux, interrogations, pistes de réflexion dans un champ de recherche éclaté ", Savoirs, 2, 2004, p. 9-69.

ASSUDE T. et al. (2010), Conditions des genèses d'usage des technologies numériques dans l'éducation, in STICEF, Vol. 17, http://sticef.univ-lemans.fr/num/vol2010/01-assude/ sticef_2010_assude_01p.pdf, date de la dernière visite : le 10 février 2012.

BERNADETTE C., PERAYA D., Transformation des regards sur la recherche en technologie de l'éducation, Bruxelles, De Boeck, 2007.

CHARAUDEAU P., "Pour une interdisciplinarité «focalisée» dans les sciences humaines et sociales ", Questions de Communication, 2010, http://www.patrickcharaudeau.com/Pour-une-interdisciplinarite.html, date de la dernière visite : le 17 octobre 2012.

DAVALLON J., «Objet concret, objet scientifique, objet de recherche », in Les sciences de l'information et de la communication: savoirs et pouvoirs, Hermès, 38, 2004, p. 30-37.

DEPOVER C., La recherche en technologie éducative : un guide pour découvrir un domaine en émergence, Paris, Agence universitaire de la francophonie, 2009.

GONNET J., Education aux médias : les controverses fécondes, Paris, Hachette, 2001.

JACQUINOT-DELAUNAY G., L'école devant les écrans, Paris, 1985, ESF.

JACQUINOT-DELAUNAY G., "Éducation et communication : l'épreuve des médias ", Hermès, 48, 2007, p. 164-171.

JACQUINOT-DELAUNAY G., De l'éducation aux médias aux médiacultures : faire évoluer théories et pratiques, 2011, INA-Sup, http://www.ina-sup.com/node/1579 date de la dernière visite : le 4 septembre 2011.

JOUËT J., « Des usages de la télématique aux Internet Studies », in DENOUËL J., GRANJON F. dir., Communiquer à l'ère numérique. Regards croisés sur la sociologie des usages, Presses des Mines, 2011, p 45-90.

LE MAREC J., "Situations de communication dans la pratique de recherche : du terrain aux composites ", Etudes de communication, 25, 2002, http://edc.revues.org/ index831.html, date de la dernière visite : le 4 avril 2012.

MIÈGE B., «L'ingénierie éducative d'un point de vue communicationnel », Distances et savoirs, 1(2), 2004, p. 129-132. 
MOEGLIN P., Outils et médias éducatifs : une approche communicationnelle, Grenoble, Presses universitaires de Grenoble, 2005.

MOEGLIN P., Une approche transdisciplinaire des outils et médias éducatifs, in 17ème Congrès SFIC 2010, http://tabarqa.u-bourgogne.fr/outils/OconfS/index.php/SIC/ SFSIC17/paper/view/144/86, date de la dernière visite : le 5 avril 2012.

MOEGLIN P., Les industries éducatives, Paris, PUF, 2010.

PASTRE de B. et al., Pour une histoire de l'audiovisuel éducatif (1950-2000), 2007, BnF. http://bbf.enssib.fr/consulter/bbf-2008-01-0097-007, date de la dernière visite : le 5 avril 2012.

PERRIAULT J., Education et nouvelles technologies: théorie et pratiques, Paris, Nathan, 2002.

PERRIAULT J., La logique de l'usage, Paris, L'Harmattan, 2008.

PERRIAULT J., Racines oubliées des sciences de la communication, Paris, CNRS éd., 2010.

PIETTE J., Éducation aux médias et fonction critique, Paris, l'Harmattan, 1996.

Résumé : Pour certains auteurs, l'étude des outils et médias éducatifs constitue un véritable champ de recherche similaire à une «interdiscipline». Si les logiques «transdisciplinaires » sont manifestes, il nous semble intéressant d'appréhender les productions de ce champ en dépassant les cadres disciplinaires. Nous avons donc choisi de mettre ces différents travaux en regard de l'attention qu'ils portent sur l'apprenant, les contenus, les technologies et le dispositif de manière plus générale. Chacune de ces approches révèle des méthodologies spécifiques, ainsi que des parti-pris épistémologiques propres. L'opposition entre démarche qualitative et quantitative, par exemple, nous éclaire quant à ces enjeux-là. Enfin, ce travail de synthèse nous permet ici de positionner notre objet de recherche afin de l'inscrire dans une perspective résolument communicationnelle.

Mots-clés : Outils et médias éducatifs, interdisciplinarité, TICE, numérique.

\begin{abstract}
For some authors, the study of educational tools and media is a search field similar to an interdisciplinary one. If the "transdisciplinary " approach is clear, it's interesting to understand the production of this field beyond these disciplinary frameworks. Therefore, we have chosen to work these next to their focus on the learner, the content, the technology and the device overall. Each of these approaches reveals specific methodologies, as much as their proper epistemological choices. The opposition between quantitative and qualitative approaches, for instance, sheds light on these issues. Finally, this overview allows us to position our study in a resolutely communicational prospect.
\end{abstract}

Keywords : Educationnal tools, ICT for education, Digitalization. 